\title{
VELOCITY REDUCTION - A POINT IN VELOCITY MODEL BUILDING FOR PRE-STACK KIRCHOFF DEPTH MIGRATION
}

DOI: http://dx.doi.org/10.18509/GBP.2015.15

UDC: 550.344.094.5 :550.83.082.13

\author{
Dr. Maya Grigorova \\ University of Mining and geology “St. Ivan Rilski”, Bulgaria
}

\begin{abstract}
Building a depth-velocity model is an important step in the pre-stack Kirchoff depth migration workflow. Conventional velocity analysis methods generally assume flatlayered geology and mild lateral velocity variations. In areas with complex structural geology formations, these methods often fail, and more sophisticated techniques are required. One possible and useful point could be velocity reduction (in percent) for finding the boundaries of the velocity model changing.

Keywords: Velocity model, velocity analysis, seismic tomography, pre-stack depth migration (PSDM)

\section{INTRODUCTION}

Migration needs a model of the wave propagation velocities of the subsurface, but obtaining this model is often the most difficult processing step in areas of complex structure. The velocity analysis method of this work combines elements of conventional velocity analysis and uses pre-stack depth migration to estimate interval velocities in structurally complex areas when lateral velocity variation is strong.

The first step of the velocity analysis procedure is to depth migrate the data with an estimate of the interval velocity model. Then apply RMO to get better summation of the migrated gathers. The output velocity model serves as the objective function for an iterative inversion of the model to estimate a perturbation to the interval velocity model that explains the observed moveout. Then depth migrate the data with the new interval velocity model and check for remaining residual moveout. Further iterations attempt to remove remaining residual moveout and correct the interval velocity model.
\end{abstract}

\section{VELOCITY MODEL REDUCTION}

To find the boundaries of the velocity model changing is used velocity reduction by percentage. For experimental purposes four velocity models are created (Fig. 1):

- Velocity model reduced with 30\% - Velocity_70\%;

- Velocity model reduced with 20\% - Velocity_80\%;

- Velocity model reduced with $10 \%$ - Velocity_90\%;

- Original velocity model without reductions - Velocity_100\%. 


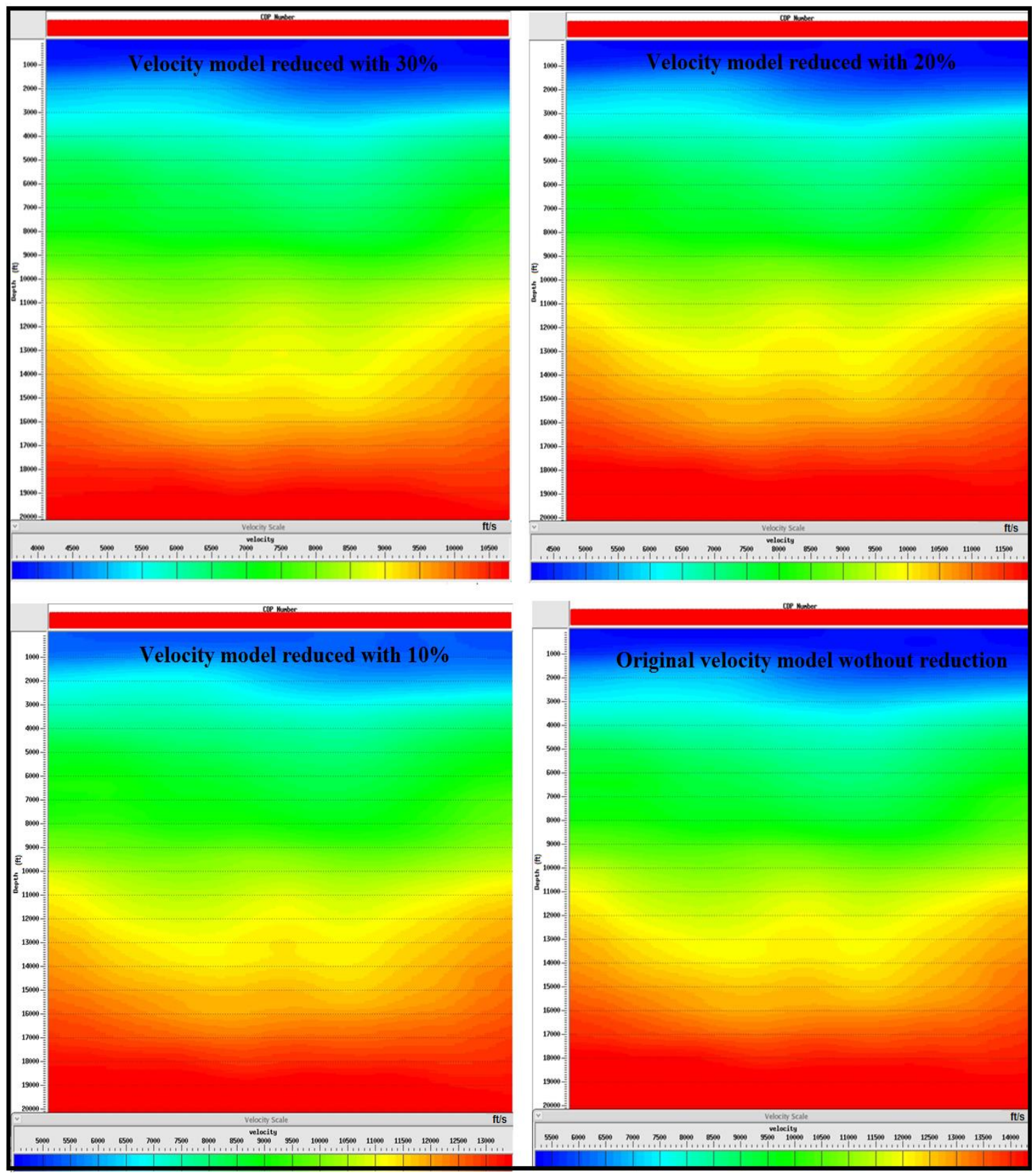

Figure 1. Velocity models: Velocity model reduced with $30 \%$, Velocity model reduced with $20 \%$, Velocity model reduced with $10 \%$, Original velocity model without reductions.

After preliminary depth migration with these velocity fields the most representative one is chosen for redefine and detailisation. Pre-Stack Depth Migration (PSDM) requires an accurate interval velocity model to achieve optimum results. The process requires utilisation of RMS velocities from the seismic data. The derived velocity field is interpolated and smoothed using proprietary software. This ensures that both the seismic data and velocity fields tie following the migration process [1]. 


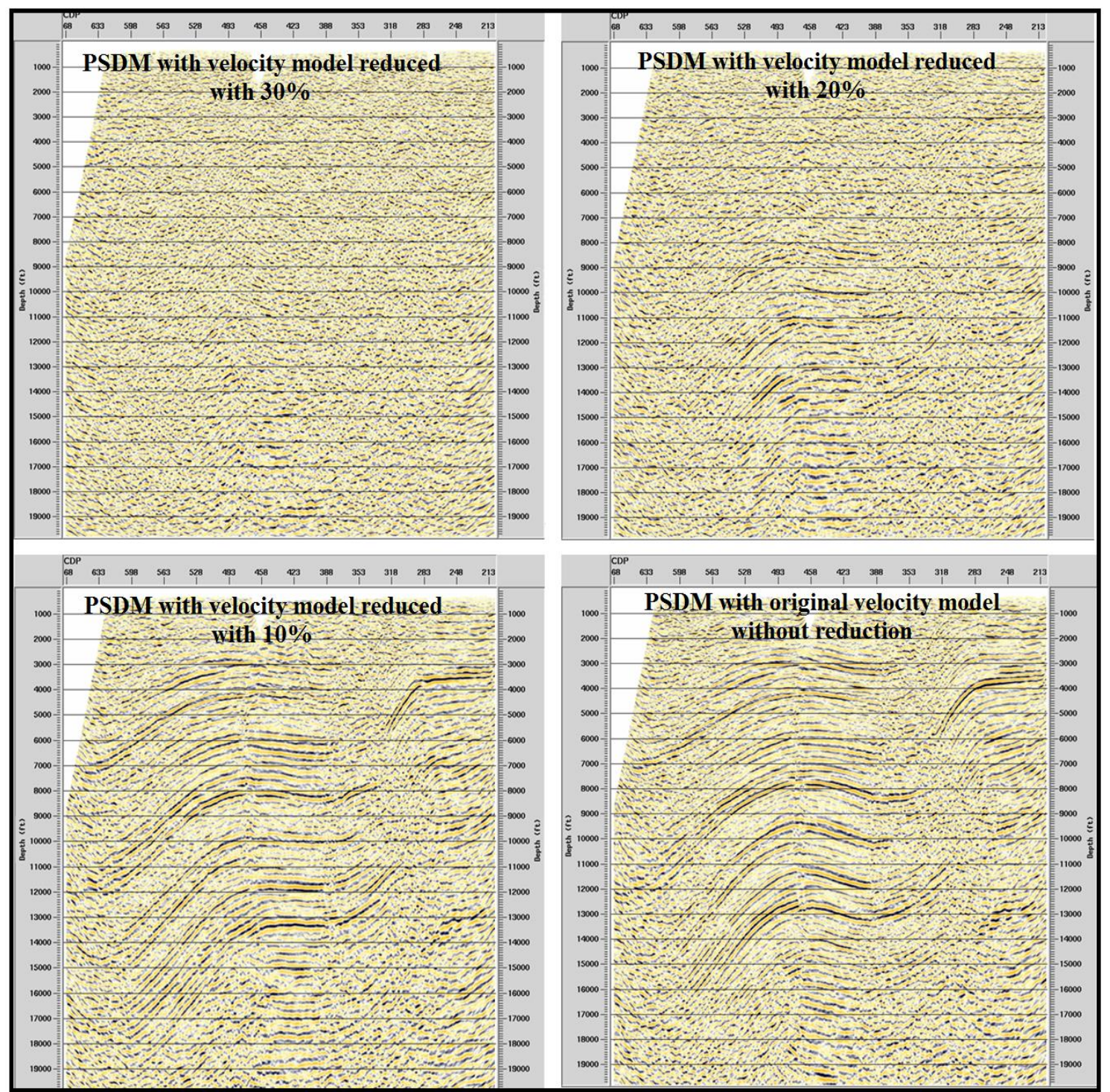

Figure 2. Preliminary pre-stack depth migrations with the following velocity models: Velocity model reduced with $30 \%$, Velocity model reduced with $20 \%$, Velocity model reduced with $10 \%$, Original velocity model without reductions.

On figure 2 is obvious that velocity models reduced with $30 \%$ and with $20 \%$ are not suitable for detailisation, because the reduction is too large and the moveout applied does not solve the velocity - reflector-depth ambiguity problem. PSDM with velocity model reduced with $10 \%$ is the most representative one, which can be observed on the edge of the stack data, where the dips are better presented, than the PSDM with original velocity model without reduction.

For following procedures on detailisation is chosen velocity model reduced with $10 \%$.

\section{DEPTH MIGRATION AND VELOCITY MODEL DETAILISATION}

Most interval-velocity estimation methods are based less ormore on traveltime tomography. Conventional traveltime tomography uses the traveltimes of picked events and ray tracing to find a velocity model and reflector position that agree with the picked traveltimes [2]. The velocity - reflector-depth ambiguity problem [3] makes solving for both velocities and reflector depth difficult. To get more coherent images of reflectors, it 
is possible to be used traveltime tomography combined with depth migration to estimate the velocity models [4]. Using depth migration makes reflector-depth location easier and more stable, but does not solve the greatest drawback of those approaches, the extensive traveltime picking required.

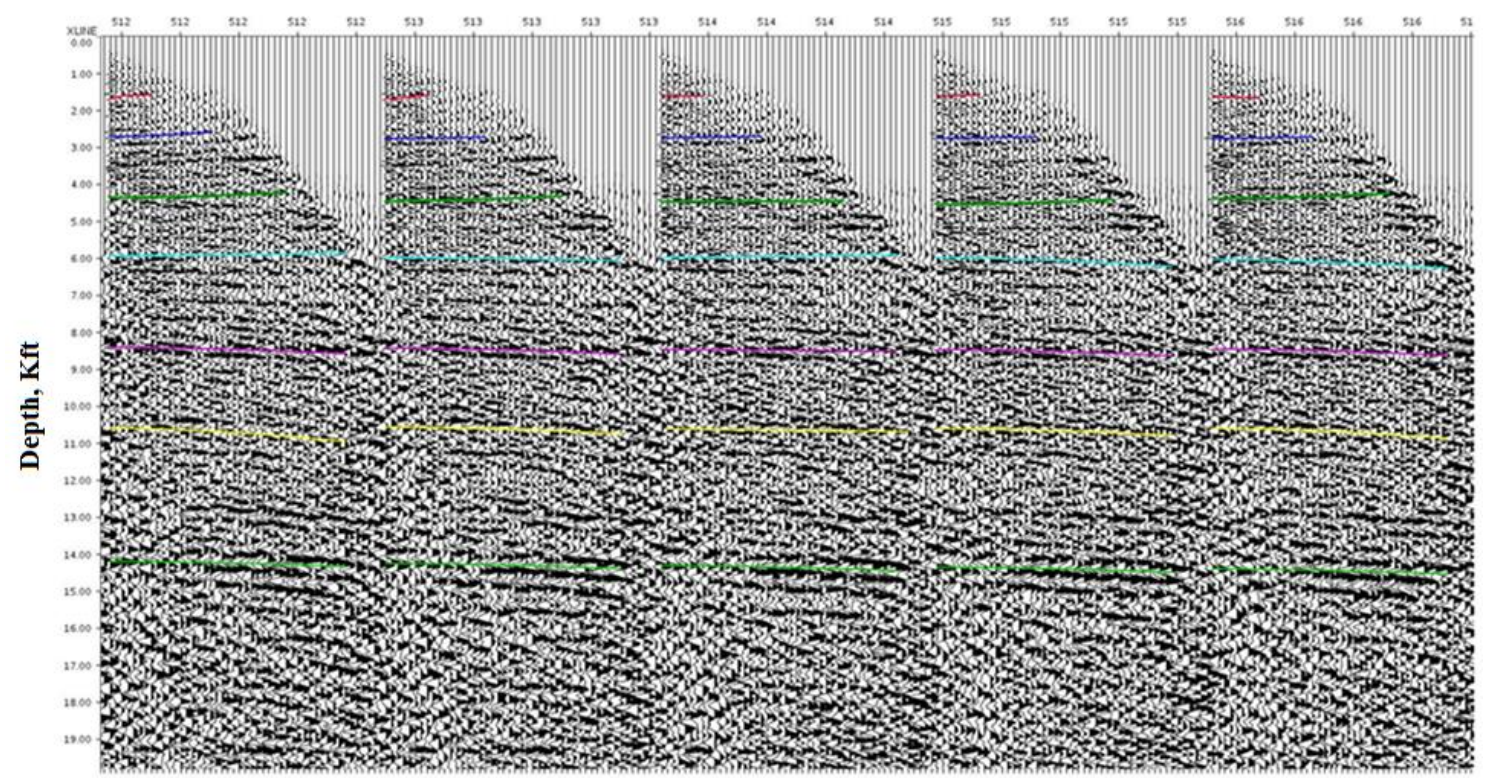

Figure 3. Traveltime picking on depth migrated gathers

Picking is labor intensive and prone to errors and biased by the interpreter. Sometimes the extra knowledge by picking is a great benefit, but then it is hard to tell how much the velocity model is constrained by the data and how much it is just invented by the interpreter.

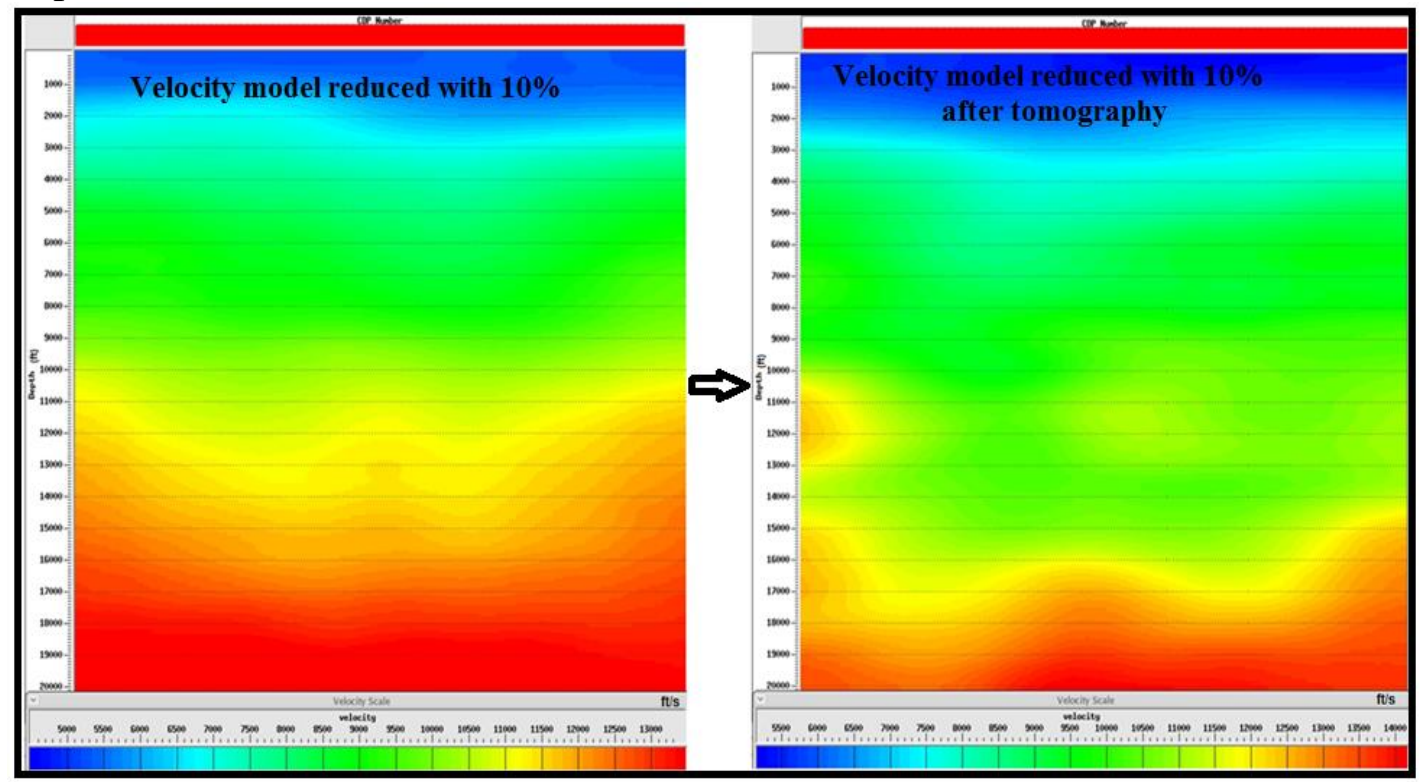

Figure 4. Comparison between velocity model reduced with $10 \%$ from original one and tomography based velocity model with reduction of $10 \%$ from original one

On figure 4 is shown comparison between velocity model reduced with $10 \%$ from original one and tomography based velocity model with reduction of $10 \%$ from original one. It is visible that the tomography based velocity model is far more precise and detailed. 


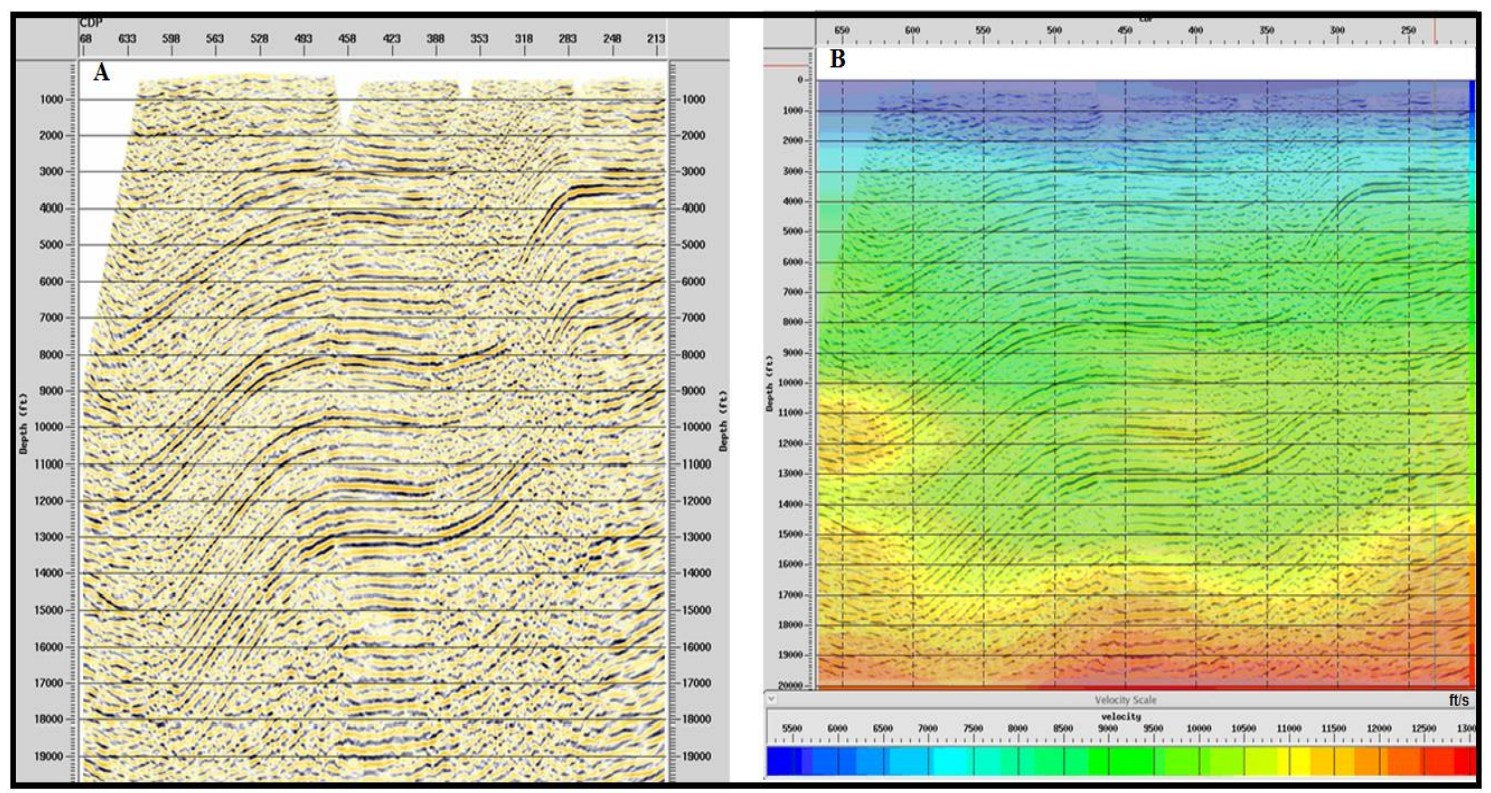

Figure 5. A - Stack data after PSDM with velocity model reduced with $10 \%$ after tomography; B -

Velocity field reduced with $10 \%$ after tomography, overlay by the stack data after PSDM

From figure 5 is obvious that the velocity model obtained by tomography fits better to reflections on the stack data, which is overlay the velocity field.

\section{CONCLUSION}

Migration should be a part of velocity estimation since an accurately migrated image is what we hope to obtain from detailed velocity analysis. Demonstrated technique is useful tool for fast estimation of velocity model variations. It provides valuable information for lateral velocity changing and can be used as a first step in precise velocity model building for pre-stack depth migration. Result from pre-stack depth migration obtained by these method combined with tomography is reliable and stable, that why can be used successfully for structural interpretation purposes.

\section{REFERENCES}

[1] Grigorova, M., Time - to - Depth migration using tomography based velocity model, 7th Congress of Balkan Geophysical Society - Tirana, Albania, 7-10 October, 2013, No 18499 ;

[2] Bishop, T. N., Bube, K. P., Cutler, R. T., Langan, R. T., Love, P.L., Resnik, J. R., Shuey, R. T., Spindler, D. A., Wyld, H. W., Tomographic determination of velocity and depth in laterally varying media. Geophysics, 50, 1985, pp 903-923;

[3] Stork, C., and Clayton, R. W., Applications of tomography to two data sets containing lateral velocity variations, 57-th Ann. Internat. Mtg., Soc. Expl. Geophys., Expanded Abstracts, 1987, pp 839-842;

[4] Stork, C. Ray trace tomographic velocity analysis of surface seismic reflection data: Ph.D. thesis, California Institute of Technology, 1988; 
International Scientific Conference GEOBALCANICA 2015 